\title{
Preparation and Properties of Epoxy Resin-Coated Micro-Sized Ferrosilicon Powder
}

\author{
Jiangang Kü ${ }^{a *}$, Huihuang Chen ${ }^{b}$, Kui He ${ }^{a}$, Quanxiang Yan ${ }^{a}$ \\ ${ }^{a}$ College of Zijin Mining, Fuzhou University, Fuzhou 350116, Fujian, China \\ ${ }^{b}$ School of Chemical Engineering, University of Queensland, St. Lucia, Brisbane, QLD 4072, Australia
}

Received: October 27, 2015; Revised: February 3, 2016; Accepted: June 13, 2016

\begin{abstract}
Ferrosilicon powder surface coated with a dense epoxy resin membrane was prepared via coating precipitation methods using silane coupling agents as the modifier and epoxy resin as the coating agent. FTIR, FESEM, MPMS-XL, and TG-DSC were used to analyze the morphology, surface composition, magnetic property and thermostability of ferrosilicon powder before and after the modification and coating. The experimental results indicate that epoxy resin membranes of a certain thickness were successfully coated onto the surface of ferrosilicon powder; coatings of epoxy resin contributed to the decreased the rate of weight loss via the reduced wear of the coatings and provided resistance to corrosion; the apparent viscosity of medium suspension with coated ferrosilicon was smaller than that of magnetite. Meanwhile, analysis reveals that room-temperature magnetic hysteresis loops of ferrosilicon powder remain basically unchanged before and after coating.
\end{abstract}

Keywords: ferrosilicon powder, epoxy resin coating, viscosity, magnetic hysteresis loops, oxidation resistibility

\section{Introduction}

Heavy liquids and/or heavy suspensions with densities between those of valuable minerals and gangue minerals are adopted as the media to achieve the objective of separation in the dense medium separation (DMS) process ${ }^{1}$. DMS includes heavy-fluid separation and suspension liquid separation. Weighting agents, which are particularly important as a kind of suspension liquid separators, were used in heavy medium separation by Blezard et al. ${ }^{2}$ and Bradbury et al. ${ }^{3}$ respectively. DMS is a process whereby particles are sorted primarily on the basis of their densities. Particles with a wide range of densities are introduced into a medium suspension of a given density. Those particles that are lighter than the medium density rise. These are commonly referred to as floats. The particles that are heavier than the medium density sink, and these are commonly referred to as sinks ${ }^{4}$. In industrial production, considering the required separating density, viscosity and stability of the system, the selected weighting agents should have high density, be difficult to make muddy and to oxidize, and be easy to recycle 5 . In principle, media with a density higher than that of water can be used as the weighting agents ${ }^{6}$. Currently, the commonly used weighting agents in industry are magnetite, ferrosilicon, ilmenite, barite, pyrite and industrial waste, such as reduced iron and magnetic beads, which were industrially used by Hamidreza et al. ${ }^{7}$ and experimentally used by Ramadan et al. ${ }^{8}$ respectively.

Magnetite is widely used in dense medium coal separation due to its low cost. However, magnetite has the following limitations compared with ferrosilicon. First, it is difficult to control the particle-size distribution of magnetite powder, which results in the huge reduction of density distribution uniformity of the suspension system, which is one of the most important technical indicators for $\mathrm{DMS}^{9}$. Second, the rheological properties of the suspension system prepared by magnetite powder are poor

* e-mail: kkcc22@163.com.cn because magnetite powder is nonspherical. The $\tau$ values and $\eta$ values of the suspension systems prepared using magnetite powder are far greater than those prepared using ferrosilicon powder under the same system density (above $\left.2.34 \mathrm{~g} / \mathrm{cm}^{3}\right)^{10,11}$. Third, magnetite has edges and corners; as a result, suspension systems prepared using magnetite will intensify the wear of the pipelines and equipment ${ }^{12,13}$. Fourth, magnetite powder is brittle, and the number of coarse particles will decrease because of the wear and tear ${ }^{14}$. Correspondingly, the number of fine particles will increase. Hence, it is difficult to achieve the automatic control of the density of the suspension system ${ }^{15}$. Fifth, the density of magnetite (approximately $4.2 \mathrm{~g} / \mathrm{cm}^{3}$ ) is smaller than that of ferrosilicon; therefore, more magnetite will be required to prepare a suspension system of the same density with respect to ferrosilicon.

However, the poor antioxidant capacity and high price limit the application range of ferrosilicon. Specifically, spray ferrosilicon of high density is easy to oxidize in aqueous solutions, and the presence of carbon impurities will enhance the oxidization rate, resulting in faster wear ${ }^{16}$. Therefore, understanding how to improve the antioxidant capacity is of crucial importance.

In this paper, the preparation of epoxy resin-coated ferrosilicon powder is described. The antioxidant capacity of such powder is studied using FTIR and FE-SEM, and the room-temperature magnetic hysteresis loops and thermostability before and after modification are analyzed by MPMS-XL and TG-DSC, respectively.

\section{Experimental Section}

\subsection{Materials and reagents}

The epoxy resin 618 and epoxy resin curing agent 5618 were obtained from Shanghai Resin Factory Co., Ltd. (Shanghai, 
China). The epoxy resin 618 is a kind of bisphenol A-type epoxy resin with the Epoxy value $0.51 \sim 0.54 \mathrm{eq} / 100 \mathrm{~g}$ and the curing agent 5618 is a modified aliphatic amine containing aromatic ring adduct containing 260-300 $\mathrm{mgKOH} / \mathrm{g}$ amine. The KH-550 silane coupling agent was obtained from Beijing Shenda Fine Chemical Company (Beijing, China); its structure is:

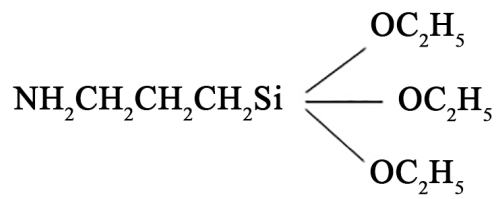

The reagent-grade ethyl alcohol (alcohol content: 99.7\%) was obtained from Tianjin Jinhuitaiya Chemical Reagent Co., Ltd. (Tianjin, China). The soft magnetic metal powder of ferrosilicon (nitrogen atomized powder, Fe 92 93, Si 5 6, Cr 1 2, and other impurities less than 0.1 wt.\%, density 7.35 $\mathrm{g} / \mathrm{cm}^{3}$ ) was obtained from Changsha Tianjiu Metal Materials Co., Ltd. (Changsha, China). The deionized water used in the experiment was lab-made.

\subsection{Analysis and characterization}

An infrared spectrometer, Nicolet 380, was used to study the FTIR spectra of ferrosilicon particles to determine whether new chemical bonds are generated before and after modification via silane coupling agent and coating with epoxy resin and to further analyze the effects of modification and coating. A Nova NanoSEM 230 model Scanning Electron Microscope was used to analyze the morphology and coating thickness of the epoxy resin-coated ferrosilicon powder. MPMS (SQUID)-XL measurement was carried out to determine the magnetic property before and after the modification and coating. A simultaneous thermal analyzer Netzsch STA449C was used to analyze the antioxidation capacity of ferrosilicon powder before and after coating. All other experimental equipment used included an electronic analytic balance BSA822, a precise timing electric mixer JJ-1, an ultrasonoscope VGT-1730TD, a thermostatic water bath DF-101S and a vacuum drying oven DZF-6020.

\subsection{Methods}

The pretreatment experiments were first performed by taking $5 \mathrm{~g}$ of ferrosilicon powder $(1.25 \pm 0.074 \mathrm{~mm})$ into a certain volume of ethanol medium for ultrasonic treatment for $15 \mathrm{~min}$ to obtain the pretreated ferrosilicon powder. Then, $80 \mathrm{ml}$ of absolute ethanol, $5 \mathrm{ml}$ of deionized water, and $2.0 \mathrm{~g}$ of silane coupling agent were collected, blended and stirred at low stirring speed for $0.5 \mathrm{~h}$ for the hydrolysis of silane coupling agent. Next, the pretreated ferrosilicon powder was placed into the hydrolysate and immersed for 0.5 h. Subsequently, $1.5 \mathrm{~g}$ of mixture of epoxy resin and epoxy resin curing agent was placed into the above solutions, stirred for $3 \mathrm{~h}$, and then separated by filtration, followed by washing by deionized water three times. The mixture was then dried and solidified at $100{ }^{\circ} \mathrm{C}$ in a vacuum drying oven for $1 \mathrm{~h}$.

After layer-by-layer coated for 10 times according to the above procedure, the following procedure was used.
Eighty milliliters of absolute ethanol was blended with $3.0 \mathrm{~g}$ of a mixture of epoxy resin, epoxy resin curing agent and ferrosilicon particles pretreated with ultrasonic in ethanol for $15 \mathrm{~min}$. Next, the pretreated ferrosilicon particles was placed into the hydrolysate and stirred for $3 \mathrm{~h}$, after which ferrosilicon powder was separated by filtration, washed by deionized water three times, and then dried and solidified at $100{ }^{\circ} \mathrm{C}$ in a vacuum drying oven for $1 \mathrm{~h}$. The steps described above were repeated to obtain epoxy resin-coated ferrosilicon powder for coating times higher than 10 .

\section{Results and Discussion}

\subsection{Effect of coating times on the morphology of epoxy resin-coated ferrosilicon powder determined by analysis of FE-SEM images}

The coating times and morphology of epoxy resincoated ferrosilicon powder are shown in Figure 1. The surface morphologies of the bare and the coated ferrosilicon powder with different coating times and magnifications are shown. Ferrosilicon particles share the characteristics of an irregular drop shape and basically take the form of spheres with relatively smooth and non-concave/convex surfaces. Furthermore, the degree of sphericity of coated ferrosilicon particles with different coating times almost remains unchanged compared to that of the uncoated particles. However, the surface smoothness decreases with decreasing coating times, especially when the coating times are higher than 25 . Hence, all further experiments were performed with coating times of 25 .

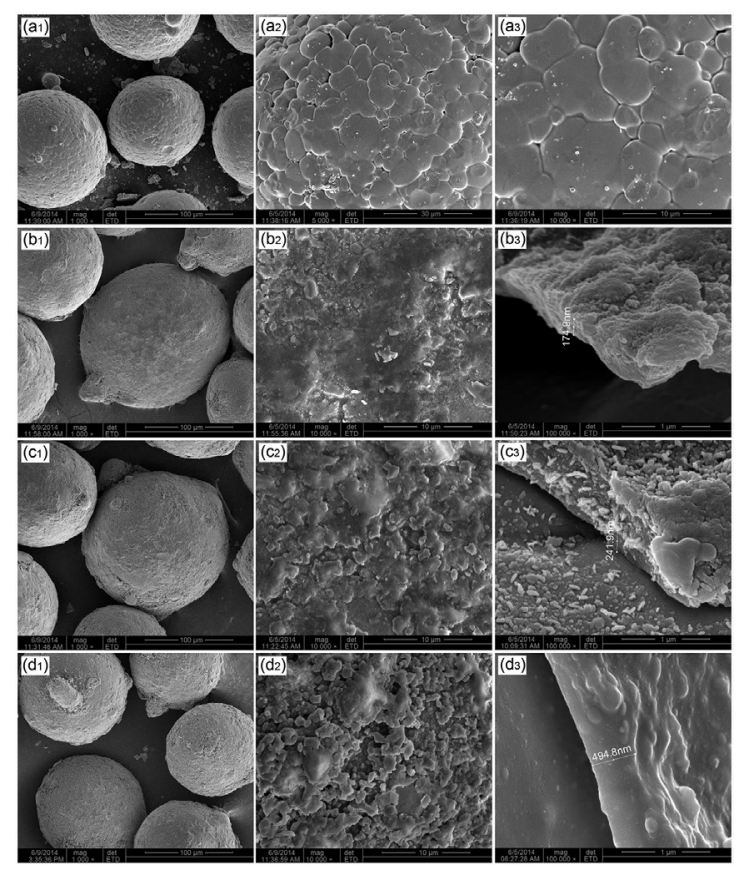

Figure 1: FE-SEM images of ferrosilicon particles: (a) uncoated, (b) coated 20 times, (c) coated 25 times, and (d) coated 40 times at different magnification 
Figure 2 shows that the thickness of the epoxy resin coated onto the ferrosilicon powder surface increases with the increase in the coating times. In addition, the crosssectional images (Figure 1) at high resolution demonstrate that epoxy resin membranes coated onto the ferrosilicon powder surface are smooth and have a compact structure without holes and stratification phenomenon, which indicates that chemical reactions occurred between layers of epoxy resin and that the layers of epoxy resin were held together by chemical bonds.

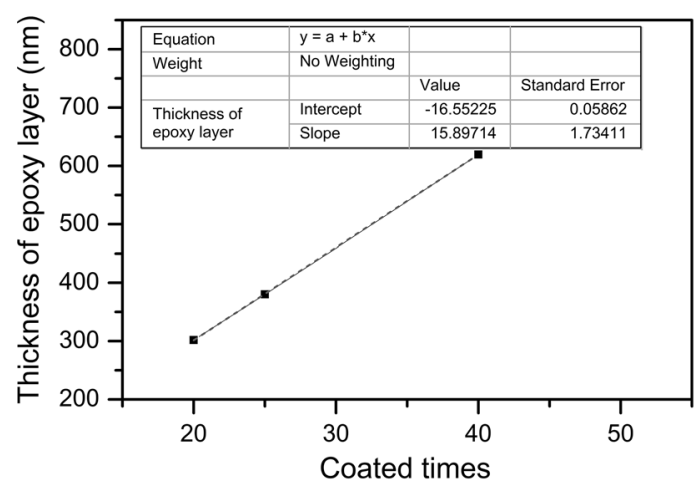

Figure 2: Relationship between the thicknesses of the epoxy resin and the number of coatings

Figure 2 shows that the thickness of the epoxy resin coated onto the ferrosilicon surface increases linearly with increasing coating times. Specifically, the mean thickness of the epoxy resin film increases $15 \mathrm{~nm}$ with each coating.

\subsection{Effect of modification and coating on the FTIR spectra of the ferrosilicon particles}

FTIR spectra (background corrected) of ferrosilicon particles before and after modification and coating are shown in Figure 3. As observed in the FTIR spectra, peaks at 3435 $\mathrm{cm}^{-1}$ and $1634 \mathrm{~cm}^{-1}$ attributed to $\mathrm{O}-\mathrm{H}$ stretching vibration and flexural vibration, respectively, are associated with the constitution water and the free water. The peak at $1457 \mathrm{~cm}^{-1}$ in the FTIR spectra of the modified ferrosilicon particles by the silane coupling agent results from the generated silanol $\left(\mathrm{NH}_{2}\left(\mathrm{CH}_{2}\right)_{3} \mathrm{SiOH}\right)$. The bands between $1450 \mathrm{~cm}^{-1}$ and 1350 $\mathrm{cm}^{-1}$ are attributed to the deformation vibrations of $-\mathrm{CH}_{2}$. The bands at $1100 \mathrm{~cm}^{-1}$ and $460 \mathrm{~cm}^{-1}$ are attributed to the $\mathrm{Si}-\mathrm{O}-\mathrm{Si}$ antisymmetric stretching vibration and flexural vibration, respectively, which demonstrates that a chemical reaction occurred during the modification. The band at 1560 $\mathrm{cm}^{-1}$ in the FTIR spectra of coated ferrosilicon particles results from the $\mathrm{C}-\mathrm{N}$ stretching vibration, the band at $1200 \mathrm{~cm}^{-1}$ corresponds to the $\mathrm{C}-\mathrm{C}$ antisymmetric stretching vibration, and the bands in the range from 800 to $860 \mathrm{~cm}^{-1}$ are attributed to bending vibration adsorption of two adjacent $\mathrm{H}$ atoms of the opposite-substituted benzene ring, indicating that the surface of the coated ferrosilicon particles is covered by epoxy resin.

Through the analysis, the modification mechanism of ferrosilicon particles by the silane coupling agent can be

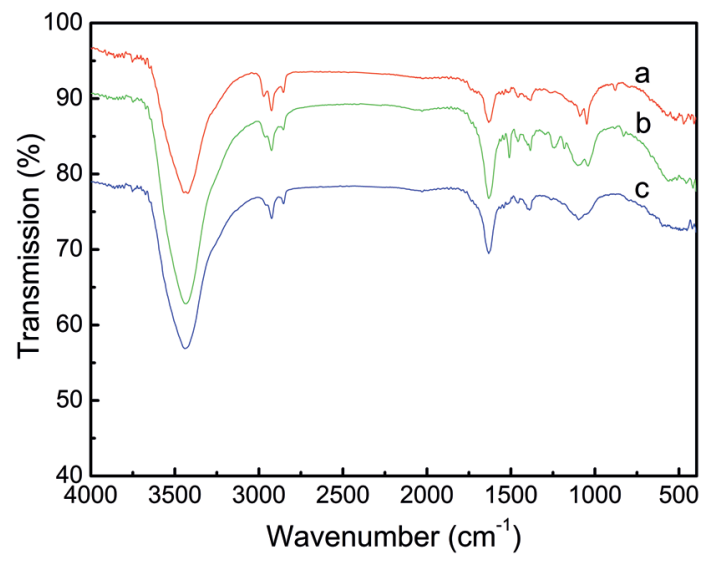

Figure 3: FTIR spectra of the ferrosilicon particles: (a) unmodified, (b) modified-coated, and (c) modified

concluded: first, hydrolysis of $\left(-\mathrm{OCH}_{2} \mathrm{CH}_{3}\right)_{3}$ generates silanol $\left(\mathrm{NH}_{2}\left(\mathrm{CH}_{2}\right)_{3} \mathrm{SiOH}\right)$ when the carbonyl of the silane coupling agent $\mathrm{KH}-550$ encounters hydroxy created by water near the surface of the ferrosilicon particles. Next, hydrogen bonds between part of the silanol and the carbonyl near the surface of the ferrosilicon particles form or further condense to form $\mathrm{Si}-\mathrm{O}-\mathrm{Fe}$. Meanwhile, the silanol groups of the silane coupling agent interact to produce a condensation product to form the net structure on the surface of ferrosilicon particles. There is a large amount of $-\mathrm{NH}_{2}$ groups in the $\mathrm{KH}-550$ modified ferrosilicon particles that can react with the epoxy groups of 618 epoxy resin to form epoxy resin membranes.

\subsection{Magnetic property measurement}

Room-temperature magnetic hysteresis loops of ferrosilicon particles before and after modification and coating are shown in Figure 4. The magnetic hysteresis loops of three types of ferrosilicon particles (unmodified, modified and coated) are narrow and smooth and share the single soft magnetic property. Additionally, the coercivity and remanence of ferrosilicon particles before and after modification almost remained the same, and only the coercivity and the remanence of the coated ferrosilicon particles decreased $0.3 \%$, which is partially due to the presence of the epoxy resin coating. In conclusion, magnetic hysteresis loops of ferrosilicon particles almost remain unchanged before and after coating, and its magnetic properties remained intact ${ }^{17}$.

\subsection{Thermostability measurement}

Simultaneous thermogravimetry (TG) and differential scanning calorimetry (DSC) experiments were carried out before and after coating with epoxy resin using a Netzsch STA $449 \mathrm{C}$ Jupiter at a heating rate of $10^{\circ} \mathrm{C} \mathrm{min}^{-1}$. The results are shown in Figure 5.

As observed from Figure 5(a), the first highest anodic peak of TG curve resulted from the oxidation (an exothermic process) of iron due to the formation of an iron-carbon primary battery on the surface of the ferrosilicon particles ${ }^{18}$, with the weight increase of $0.1 \%$. At the same time, evaporation of adsorbed 


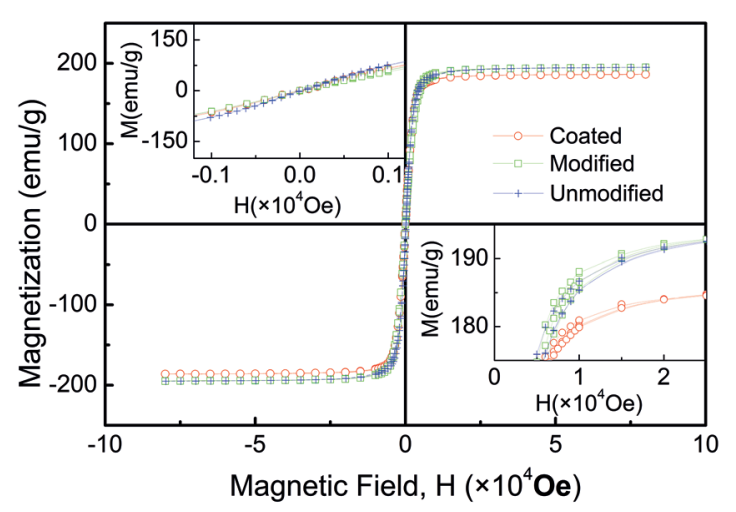

Figure 4: Room-temperature magnetic hysteresis loops of ferrosilicon particles

water on the surface of the ferrosilicon particles occurred, with a weight loss corresponding to a wide adsorption peak which can be seen from DSC curve. TG reveals the course of the weight loss at $180^{\circ} \mathrm{C}$ resulting from the oxidation of carbon. As all of the above-mentioned processes occur at the surface of ferrosilicon particles, the change in the DSC curve is attributed to the endothermic reaction.

According to Figure 5(b), there is an adsorption peak at $40{ }^{\circ} \mathrm{C}$ in the DSC curve of the coated ferrosilicon particles resulting from the evaporation of adsorbed water on the surface of ferrosilicon particles along with the weight loss. At $250^{\circ} \mathrm{C}$, an obvious adsorption peak existed with weight loss, which resulted from the structural damage of the epoxy resin. The weight leveled off after $280^{\circ} \mathrm{C}$ because the structure of epoxy resin was completely destroyed. It is clear that the antioxidant capacity of iron in the coated ferrosilicon particles was improved as no oxidation exothermic peak of iron is observed in the DSC curve of coated ferrosilicon particles.

\subsection{Wear resistance measurement}

The wear resistance experiment of coated ferrosilicon particles was performed in an Agitator (IKA RW20 mixer) as a function of time to determine the corrosion resistance ability of coated FeSi particles. The density of used ferrosilicon suspensions with tap water is $3.0 \mathrm{~kg} / \mathrm{l}$. And the stirring rate is kept constant during the experiment. Under given time intervals, coated ferrosilicon particle is sampled and characterized by SEM technique. Results are shown in Figure 6.

It is obvious from Figure 6(a) and Figure 6(b) that FeSi particles are still coated completely after $8 \mathrm{~h}$ stirring. However, corrosion of coated FeSi particles happens after 9h (Figure 6(c)). This is consistent with the weight loss rate results. The weight loss of the coated sample is only negligible $0.03 \%$ in the first $8 \mathrm{~h}$ (Figure 6(d)). Thereafter, weight loss dramatically increases up to around 3\% after $9 \mathrm{~h}$. This demonstrates that the coating is quite stable and resistant to corrosion in the first $8 \mathrm{~h}$. However, corrosion occurs when the stirring time is longer than $9 \mathrm{~h}$.

\subsection{Viscosity measurement}

Two samples of coated ferrosilicon particles and magnetite were used in the tests. The magnetite (assayed at $71.3 \% \mathrm{Fe}$ and $1.1 \% \mathrm{SiO}_{2}$ with the density $5.0 \mathrm{~kg} / \mathrm{m}^{3}$ ) provided by the Makeng Mining Co., Ltd. (Fujian, China) was studied as a comparison. Prior to laboratory experiments the samples were analyzed using an OMEC Sample Analyzer (LS-CWMIII) to define their size ranges. It can be seen in Figure 7 that the average size of ferrosilicon and magnetite is similar, which is 76.3 and $75.8\left(D_{\text {av }} / \mu \mathrm{m}\right)$, respectively. It is also observed that the particle size distribution of magnetite is broader than that of ferrosilicon.

Since the measurements were carried out to study the effect of viscosity of the different suspensions used as dense medium in gravity separation, tap water was utilized in measurements. As known, the samples are magnetic and remnant magnetic forces cause aggregation that may strongly affect the viscosity measurements. In order to reduce the negative impact and obtain good reproducibility, high-speed blending was applied to prepare the suspensions.

The viscosity tests of different suspensions were carried out using Brookfield DV- II Pro viscosimeter with ultra-low viscosity adapter at room temperature $\left(23.6^{\circ} \mathrm{C}\right)$. The results are shown in Figure 8.

It is obvious from Figure 8 that the apparent viscosity of ferrosilicon suspension is smaller than that of magnetite in the whole measurement range. The medium density plays an (a)

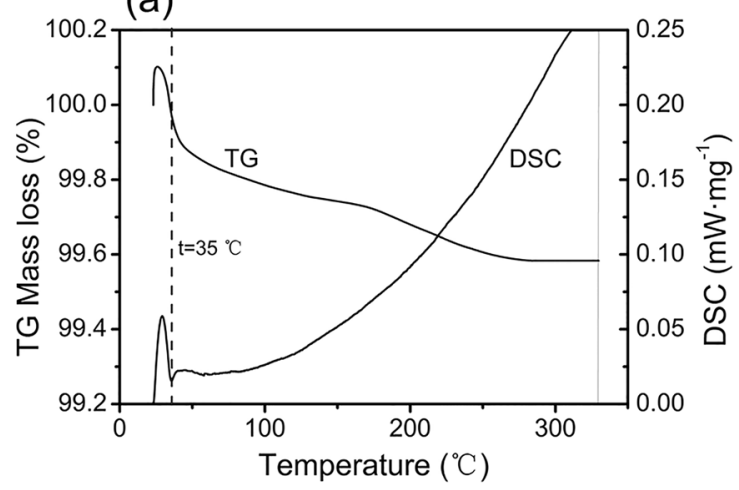

(b)

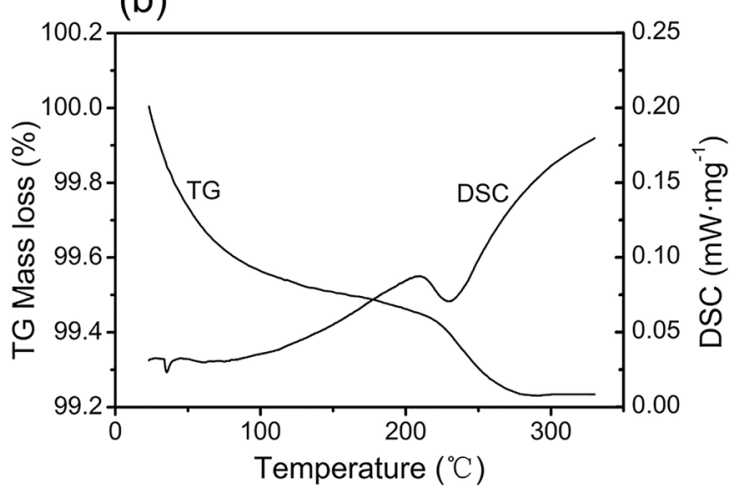

Figure 5: TG-DSC curves of ferrosilicon particles: (a) uncoated (b) coated 

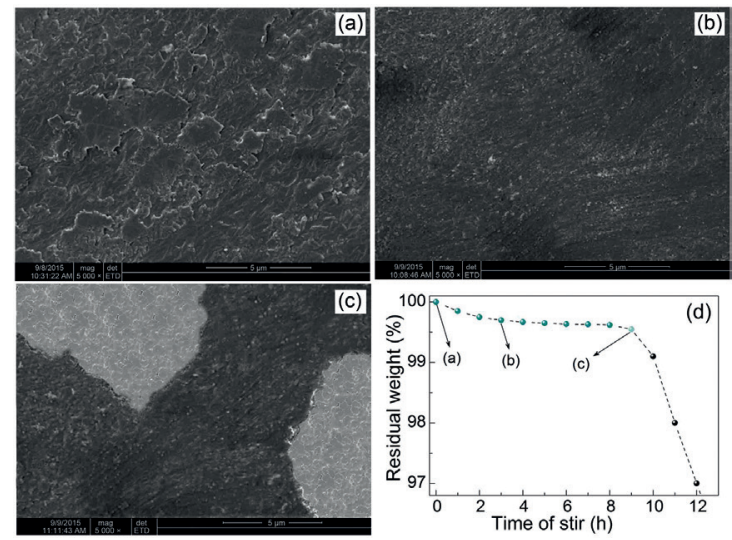

Figure 6: Effect of stirring time on residual weight of coated ferrosilicon particles. SEM micrographs of (a) $t=0 h$, (b) $t=3 h$ and $(c) t=9 h$.

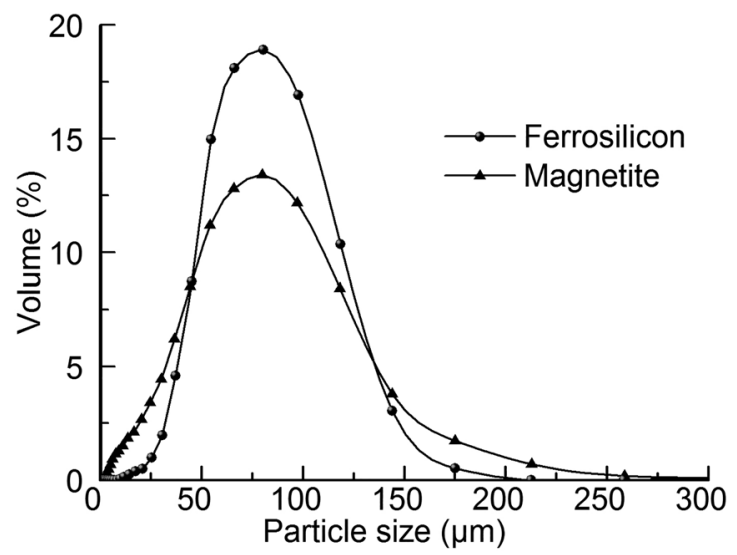

Figure 7: Particle size ranges determined for the coated ferrosilicon particles and magnetite

important role in the apparent viscosity of two suspensions, especially when the medium density is greater than $2.2 \mathrm{~kg} / \mathrm{l}$. To be more specific, the apparent viscosity of two suspensions

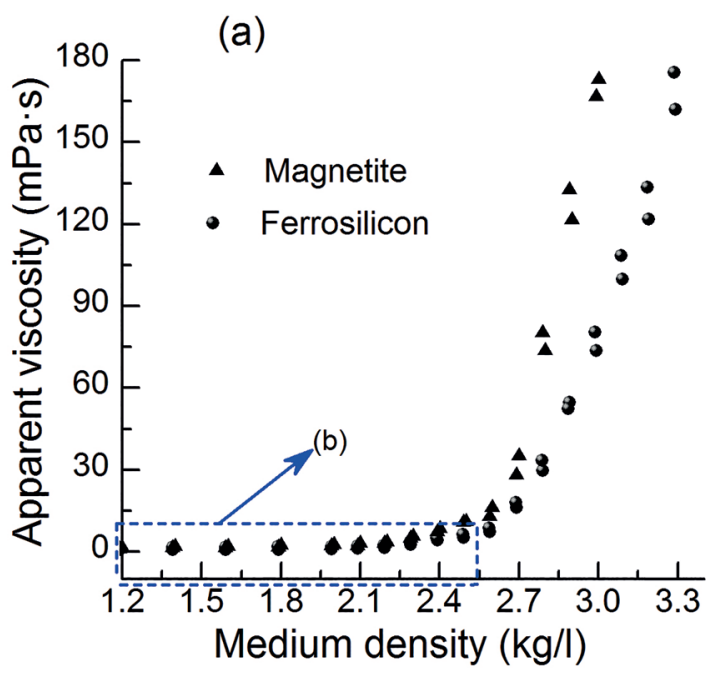

increases slowly when the medium density is from 1.2 to $2.2 \mathrm{~kg} / \mathrm{l}$ and then increases quickly along with the increase of the medium density.

\section{Discussion}

There are many advantages of coated ferrosilicon powders in practical applications. First, it is easy to control the particle-size distribution of coated ferrosilicon powders, which is one of the most important technical indicators for $\mathrm{DMS}^{19}$. Second, the rheological property of the suspension system prepared by coated ferrosilicon powders with high sphericity is good for accelerating the DMS process because the $\tau$ and $\eta$ values of the suspension systems are far lower than those of the other suspension systems under the same density (especially a density above $\left.2.34 \mathrm{~g} / \mathrm{cm}^{3}\right)^{20}$. Third, the wear and tear of pipelines and equipment will decrease sharply with the use of coated ferrosilicon powder with high sphericity $^{21}$. Fourth, the particle size characteristics of the suspension system will not change much because the wear resistance of the coated ferrosilicon powders will be greatly increased. Hence, it is easy to achieve automatic control of the density of the suspension system.

In brief, the suspension system prepared by coated ferrosilicon powders has many advantages, such as high density, high stability, good fluidity, good durability, and ease of control. Therefore, the applications of coated ferrosilicon powders will continue to expand with the development of the technology. Because the epoxy resin-coated ferrosilicon powders are mainly used in ambient atmosphere, preventing the oxidation of iron and reducing the wear rate in water are the best ways to increase the working life of ferrosilicon particles. Thus, the as-prepared ferrosilicon particles coated by epoxy resin can expect to address this issue.

\section{Conclusions}

In this paper, ferrosilicon powder coated with a dense epoxy resin membrane was obtained via heterogeneous

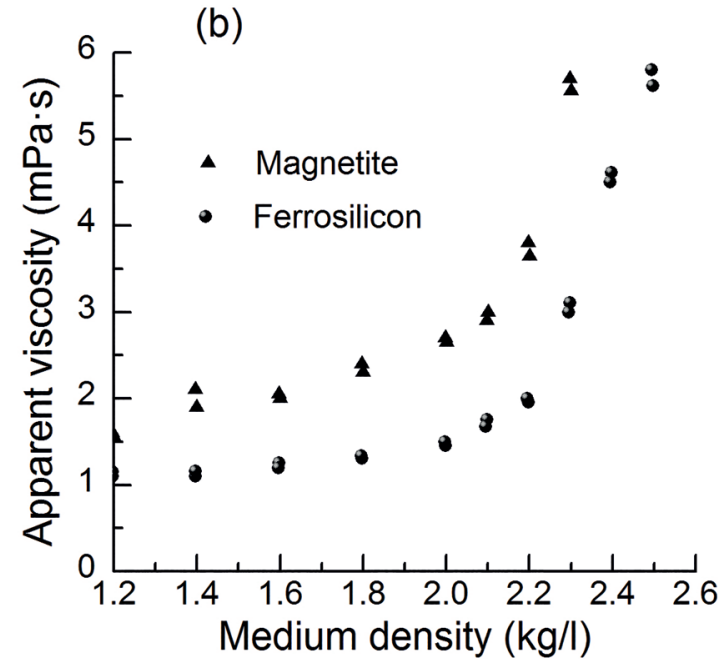

Figure 8: (a) Effect of medium composition on apparent viscosity. (b) Selected zone of (a). 
precipitation coating methods using silane coupling agents as the modifier and epoxy resin as the coating agent.

The thickness of ferrosilicon particles increases by approximately $10 \mathrm{~nm}$ after each coating, and ferrosilicon particles remain spherical after coating 20 times, without aggregation among particles. The FT-IR results reveal that coating of the silane coupling agent KH550-modified ferrosilicon particles by epoxy resin is attributed to chemisorption.

The experimental results indicate that epoxy resin membranes of a certain thickness were successfully coated onto the surface of ferrosilicon powder; coatings of epoxy resin contributed to the decreased the rate of weight loss via the reduced wear of the coatings and provided resistance to corrosion; the apparent viscosity of medium suspension with coated ferrosilicon was smaller than that of magnetite. Meanwhile, analysis reveals that room-temperature magnetic hysteresis loops of ferrosilicon powder remain basically unchanged before and after coating.

\section{Acknowledgements}

This work was supported by the National Natural Science Foundation of China (Grant No. 51104048), for which the authors express their appreciation.

\section{References}

1. Law HS, Masliyah JH, Mac Taggart RS, Nandakumar K. Gravity separation of bidisperse suspensions: Light and heavy particle species. Chemical Engineering Science. 1987;42(7):1527-1538.

2. Blezard M, Grover BW, Messenger ET, Nicholson WJ, Williams MJ, inventors. Albright \& Wilson Limited, assignee. Functional fluids and liquid cleaning compositions and suspending media. US Patent 5,807,810. 1998 Sep 15.

3. Bradbury A, Sawdon CA, inventors. M-I LLC, assignee. Additive for increasing the density of a fluid and fluid comprising such additive. US Patent 6,586,372. 2003 Jul 1.

4. Gupta A, Yan DS. Mineral processing design and operation: an introduction. Amsterdam: Elsevier Science; 2006.

5. Bosman J. The art and science of dense medium selection. Journal of the Southern African Institute of Mining and Metallurgy. 2014;114(7):529-536

6. Waanders FB, Rabatho JP. Recovery of heavy minerals by means of ferrosilicon dense medium separation material. In: Mercader RC, Gancedo JR, Cabral Prieto A, Baggio-Saitovitch E, eds. LACAME 2004 Proceedings of the 9th Latin American Conference on the Applications of the Mössbauer Effect (LACAME 2004) held in Mexico City, Mexico, 19-24 September 2004. Berlin Heidelberg: Springer; 2005. p. 55-60.
7. Habetinejad H, Jorjani E, Sam A. Evaluation of Mayer curve validity on feed blending at the Zarand coal washery plant. International Journal of Mining Science and Technology. 2012;22(1):19-22.

8. Ramadan Q, Samper V, Poenar DP, Yu C. An integrated microfluidic platform for magnetic microbeads separation and confinement. Biosensors and Bioelectronics. 2006;21(9):1693-1702.

9. He YB, Laskowski JS, Klein B. Particle movement in non-Newtonian slurries: the effect of yield stress on dense medium separation. Chemical Engineering Science. 2001;56(9):2991-2998.

10. Bevilacqua P, De Lorenzi L, Ferrara G. Rheology of low density suspensions in dense medium separation of post-consumer plastics. Coal Preparation. 2000;21(2):197-209.

11. Mabuza NT, Pocock J, Loveday BK. The use of surface active chemicals in heavy medium viscosity reduction. Minerals Engineering. 2005;18(1):25-31.

12. Cavenati S. Grande CA, Rodrigues AE. Upgrade of methane from landfill gas by pressure swing adsorption. Energy \& Fuels. 2005;19(6):2545-2555.

13. Chu KW, Kuang SB, Yu AB, Vince A, Barnett GD, Barnett PJ. Prediction of wear and its effect on the multiphase flow and separation performance of dense medium cyclone. Minerals Engineering. 2014;56:91-101.

14. Golden DC, Ming DW, Morris RV, Brearley AJ, Lauer HV, Treiman AH, et al. Evidence for exclusively inorganic formation of magnetite in Martian meteorite ALH84001. American Mineralogist. 2004;89(5-6):681-695.

15. Zhao ZY, Fan ZH, Zhang JJ, Xia ZQ. Research on ride comfort of nonlinear vehicle suspension. Advanced Materials Research. 2013;605-607:443-447.

16. Østensen OJ. Upgrading off-grades from the silicon process: Increasing the silicon yield from Elkem Thamshavn using mechanical or metallurgical separation. [Master Dissertation]; Trondheim: Institutt for materialteknologi NTNU; 2011.

17. Dong D, Guan J, Wang W, Li W, Zhou J. Preparation and electromagnetic properties of micrometer Fe flakes modified with epoxy resin. Acta Metallurgica Sinica. 2009;45(9):1141-1145.

18. Narayan S. Rajeshkannan A. Strain hardening behaviour in forming of sintered iron- $0.35 \%$ carbon powder metallurgy preform during cold upsetting. Materials Research. 2011;14(4):449-455.

19. Dworzanowski M. Optimizing the performance of wet drum magnetic separators. Journal of the Southern African Institute of Mining and Metallurgy. 2010;110(11):643-653.

20. Klein B. Rheology and stability of magnetite dense media $[\mathrm{PhD}$ Thesis]. Vancouver: University of British Columbia; 1992.

21. Red'kina NI, Khodakov GS, Gorlov EG. Coal fuel slurry for internal combustion engines. Solid Fuel Chemistry. 2013;47(5):306-314 . 